\title{
ADVANTAGES OF THE HAUSDORFF CENTERED MEASURE FROM THE COMPUTABILITY POINT OF VIEW
}

\author{
MARTA LLORENTE and MANUEL MORÁN*
}

(Dedicated to Professor Pertti Mattila on the occasion of his 60th birthday)

\begin{abstract}
In this paper we study Hausdorff centered measures, a useful tool in fractal geometry. The definition of Hausdorff centered measure is based on efficient coverings centered at the given set, playing a dual role to packing measures. We show that, at least in the self-similar setting, it has some advantages, from a computational point of view, over other measures based on coverings, such as the Hausdorff measure or the Hausdorff spherical measure. We also extend our results to general Hausdorff centered measures with gauge functions for which the measures scale.
\end{abstract}

\section{Introduction}

Sets with fractal dimension occur naturally in the investigation of many phenomena in pure and applied mathematics, prime among them the understanding of the chaotic behaviour of complex and real dynamical systems. The properties of such sets require the understanding of measures of fractal dimensions. The prototypical example of fractal measures are the Hausdorff measures $H^{s}$ for $s>0$. For a Borel set $A \subset \mathrm{R}^{n}$

$$
\begin{aligned}
H^{s}(A)=\lim _{\delta \rightarrow 0}\left[\operatorname { i n f } \left\{\sum\left|U_{i}\right|^{s}:\right.\right. & \left|U_{i}\right| \leq \delta, \\
& \left.\left.U_{i} \text { are convex sets such that } E \subset \cup U_{i}\right\}\right],
\end{aligned}
$$

where $|U|$ stands for the diameter of $U$.

An obstacle for the computation of $H^{s}(E)$ is that the infimum is taken over a very large class of sets.

Packing measures $P^{\theta}$ where introduced by Sullivan [27], Tricot [29] and Taylor and Tricot [28]. In [24], Saint Raymond and Tricot, introduced the so-called Hausdorff centered measures $C^{\theta}$. The packing measure has been

\footnotetext{
* This research has been supported by the Ministerio de Educación y Ciencia, research project MTM2006-02372. M. LL. was also supported by the research project CCG06-UAM/HUM-0335.

Received 4 September 2008, in final form 28 October 2008.
} 
extensively studied in the later years. In a self-similar setting, the packing measure may be easier to handle than the Hausdorff measure [23], however, it is not, in a general setting, equivalent to the Hausdorff measure in the sense that a set of null $s$-dimensional Hausdorff measure may well have an infinite $s$-dimensional packing measure. Moreover, the behaviour of the packing measure under natural operations such as projections or intersections is very irregular unlike the one of the Hausdorff (see, for example, [6], [7], [8], [9], [12], [13], [16], [19], [20].)

Much less is known about the Hausdorff centered measure, although it has attracted some attention recently (for works on the Hausdorff centered measure (= covering measure) see [3], [4], [5], [21], [25], [30], [31]). But remarkably, opposite to the packing measure, it differs by at most a constant factor from the Hausdorff measure (see (10)), so it may be used in the computation of the Hausdorff dimension.

The aim of this note is to show that the Hausdorff centered measure $C^{\theta}$ is easier to handle than the Hausdorff or Hausdorff spherical measure, at least in the self-similar setting. This makes it a more natural candidate to investigate covering-measure properties of classical fractal objects, as the Sierpinski gasket or the Koch curve. We recall that the exact Hausdorff measure of these fractal sets remains still unknown due to the complexity of the computational procedures.

Let us start with the definition given by Saint Raymond and Tricot in [24].

Definition 1. Let $\theta$ be a continuous increasing function such that

$$
\lim _{r \rightarrow 0^{+}} \theta(r)=0 \quad \text { and } \quad \limsup _{r \rightarrow 0^{+}} \frac{\theta(2 r)}{\theta(r)}<\infty .
$$

For $A \subset \mathrm{R}^{n}$ we define

$$
C_{0}^{\theta} A=\liminf _{\delta \rightarrow 0}\left\{\sum_{i=1}^{\infty} \theta\left(\left|B_{i}\right|\right)\right\},
$$

where the infimum is taken over all coverings $\left\{F_{i}\right\}$ of $A$ by balls with diameter smaller than $\delta$ and centers at $A$.

The Hausdorff centered measure $C^{\theta}$ of a set $A \subset \mathrm{R}^{n}$ is defined as

$$
C^{\theta} A=\sup \left\{C_{0}^{\theta} F: F \subset A, F \text { closed }\right\} .
$$

We will discuss first the classical case where $\theta(r)=(2 r)^{s}$, denoting the corresponding measure by $C^{s}$. Later we will analyze for which other gauge functions $\theta$ our results extend. 
The first step in Definition 1 gives hope that this measure is easier to compute than the Hausdorff measure since we take infima over a considerably smaller family of sets. On the negative side is that we need two limiting procedures in the construction. This is due to the fact that we are taking optimal coverings by centered balls and hence the resulting set function, $C_{0}^{s}$ (premeasure), is not monotone (see Example 4). This reminds of the behaviour of the packing premeasure (see (7) for a definition). Recall that the set function $P_{0}^{s}$, defined in a similar, dual, way to $C_{0}^{s}$, by replacing optimal coverings with maximal packings, is subadditive and monotone, but not $\sigma$-subadditive. Thus we must add an extra step to get a measure. However, Feng showed in [10] that if $K \subset \mathrm{R}^{n}$ is a compact set with finite packing measure, then the extra step is no longer needed.

It would be desirable to extend Feng's result to $C^{s}$. But Example 4 shows that the behavior of $C^{s}$ differs from the packing measure as it is not possible to get such an extension for general compact sets. Surprisingly, as Theorem 3 shows, on compact subsets of self similar sets satisfying the open set condition (OSC), we are able to show that the second step is not needed. The proof of Theorem 3 is based on the self-similar tiling principle proved by the second author in [23].

In [3], Edgar analyzes the properties of the Hausdorff centered measure (= covering measure) $C^{\theta}$ in general metric spaces for general gauge functions (= Hausdorff functions) $\theta$. He shows that often $C^{\theta}$ can be defined as a fine variation. That is, taking the infimum over all fine covers $\beta$ of the supremum over all packings $\pi \subseteq \beta$ of the sum $\sum_{(x, r) \in \pi}|B(x, r)|^{s}$. But this interesting result does not help in computing the exact value of a given set.

In this work (Theorem 5) we elaborate an alternative definition of the Hausdorff centered measure in the self similar setting. The point of view is entirely different from Edgar's and instead it follows the lines of [23] where similar expressions are obtained for the Hausdorff and packing measures. We first show that the Hausdorff centered measure $C^{s}(E)$ of a self-similar set $E$ coincides with its premeasure $C_{0}^{s}(E)$, making unnecessary the second step in its definition above. Then we show that the exact value of the Hausdorff centered measure in fact can be found by searching a centered ball of minimal density. Notice that the fact that the centers of the balls belong to $E$ makes easier the search of the ball of minimal density in comparison with the related result for the Hausdorff spherical measure. For the Hausdorff spherical measure the optimal balls could be centered anywhere in $\mathrm{R}^{n}$ [23]. The computation of the ordinary Hausdorff measure implies a greater degree of complexity, since it requires the search of a convex set of minimal density.

Now we turn to the issue of the general gauge function. Notice that for other metric measures when we replace $\theta(r)=(2 r)^{s}$ in the definition with an 
arbitrary gauge function $\theta(r)$, the properties of the resulting measure might be entirely different. For example, in [2] Csörnyei constructed a doubling function and a compact set for which the packing measure and premeasure do not coincide and in [25] Schechter gave an example of a gauge function of the form $\theta(x, r)=r^{t} v(B(x, r))^{q}$ where the equivalence between Hausdorff centered and spherical measure fails to hold.

In the last section we consider the general measure $C^{\theta}$ and characterize the gauge functions for which Theorems 3 and 5 extend. This extension relies on the scaling properties of $C^{\theta}$. We say that a measure $\mu$ scales (is a scaling measure) if for all $c>0$ and $A \subset \mathrm{R}^{n}$

$$
\mu(c A)=c^{s} \mu(A) .
$$

In [1] Csörnyei and Mauldin characterize those gauge functions for which the corresponding Hausdorff and packing measures scale. We prove that for the same class of gauge functions $C^{\theta}$ scales as well (Lemma 7). Then, we give a class of gauge functions so that Theorem 3 and 5 hold.

The paper is organized as follows. In Section 2 we review the needed notation and facts from fractal geometry used in the rest of the paper. In Section 3 we prove the main theorems for $C^{s}$, recalling first the self-similar tiling principle. In this section we include also an example showing that for general compact sets the two steps in the definition of $C^{s}$ are needed. Section 4 is devoted to the study of $C^{\theta}$ for general gauge functions.

\section{General facts and notations}

\subsection{Self-similar sets}

We analyze the behavior of the Hausdorff centered measure and premeasure on a self-similar set $E$ generated by a system $\Psi=\left\{f_{1}, f_{2}, \ldots, f_{m}\right\}$ of similitudes of $\mathrm{R}^{n}$ such that:

A1: $\Psi$ satisfies the open set condition (OSC), i.e., there exists an open set $\mathscr{O} \subset \mathrm{R}^{n}$ such that $f_{i}(\mathscr{O}) \subset \mathscr{O}$ for all $i \in M:=\{1,2, \ldots, m\}$ and $f_{i}(\mathscr{O}) \cap f_{j}(\mathcal{O})=\emptyset$ for $i, j \in M$ with $i \neq j$.

A2: $m \geq 2$ and $\mathrm{R}^{n}$ is the smallest linear manifold that contains $E$.

Denoting by $S \Psi$ the set mapping defined by

$$
S \Psi(X)=\bigcup_{i \in M} f_{i}(X)
$$

we can write $E=S \Psi(E)$. 
Assumption A1 ensures that the Hausdorff dimension of $E, \operatorname{dim}_{H} E$, is given by the unique real number $s$ such that $\sum_{i=1}^{m} r_{i}^{s}=1$, where $r_{i}$ is the contraction ratio of the similarity $f_{i}, i \in M$ (see [11]). Moreover, the Hausdorff measure of $E$ is finite and positive. From now on we keep this notation.

Assumption A2 avoids the case when the self-similar set reduces to a singleton and ensures that the smooth $(n-1)$-dimensional manifolds intersect $E$ in sets of null Hausdorff measure [17].

We now introduce the basic notation used below. We write $\mathcal{M}$ for the set of finite sequences of indices in $M$ plus the empty sequence $i_{0}$. Let $f_{i_{0}}=\mathrm{id}$. For $\mathbf{i}=i_{1} i_{2} \ldots i_{k} \in \mathscr{M}$, we write $f_{\mathbf{i}}$ for the similitude $f_{i_{1}} \circ f_{i_{2}} \circ \cdots \circ f_{i_{k}}$ and, for $A \subset$ $\mathrm{R}^{n}$ and $\mathbf{i} \in \mathcal{M}, A_{\mathbf{i}}$ denotes the set $f_{\mathbf{i}}(A)$ and $r_{\mathbf{i}}$ the contraction ratio of $f_{\mathbf{i}}$. For a given $q<k, \mathbf{i} \mid q$ denotes the truncation of $\mathbf{i}$ to the $q$-th place, $\mathbf{i} \mid q=i_{1} i_{2} \ldots i_{q}$. Given two sequences $\mathbf{i}=i_{1} i_{2} \ldots i_{k}$ and $\mathbf{j}=j_{1} j_{2} \ldots j_{p}$ in $\mathscr{M}$ we write $\mathbf{i} \wedge \mathbf{j}$ for the sequence $i_{1} i_{2} \ldots i_{k} \in \mathcal{M}$ with $k=\max \left\{l: i_{k}=j_{k}, 1 \leq k \leq l\right\}$ if $i_{1}=j_{1}$ and $\mathbf{i} \wedge \mathbf{j}=\mathbf{i}_{0}$ if $i_{1} \neq j_{1}$. We denote by $\mathbf{i} \vee \mathbf{j}$ the sequence $i_{1} i_{2} \ldots i_{k} j_{1} j_{2} \ldots j_{p}$ and, for $\mathscr{I}, \mathscr{J} \subset \mathcal{M}$, we write $\mathscr{I} \vee \mathscr{J}$ for the set of indices $\{\mathbf{i} \vee \mathbf{j}: \mathbf{i} \in \mathscr{I}, \mathbf{j} \in \mathscr{J}\}$. Lastly, for $\mathscr{I} \subset \mathcal{M}$ and $A \subset \mathrm{R}^{n}, \mathscr{I} A$ denotes the union $\bigcup_{\mathbf{i} \in \mathscr{I}} A_{\mathbf{i}}$.

We recall some basic properties of self-similar sets satisfying the open set condition which are used in our arguments.

In Euclidean spaces, the OSC is equivalent to the strong open set condition [26]. This means that we may assume that $\mathcal{O} \cap E \neq \emptyset$. Furthermore, if $\partial$ denotes the boundary topological operator on $\mathrm{R}^{n}$, it may be proved (see [22], Theorem 3.3) that the Hausdorff dimension of $E \cap \partial \mathscr{O}$ is strictly smaller than $s$, and therefore, $H^{s}(E \cap \partial \mathscr{O})=0$. It is easy to see that $E \subset \overline{\mathcal{O}}$ [11].

A useful measure on a self-similar set is the sometimes called natural probability measure, or Hausdorff normalized measure $\mu$, defined on the ring of cylinder sets by $\mu\left(E_{\mathbf{i}}\right)=r_{\mathbf{i}}^{s}$, and then extended to Borel subsets of $E$. This is a probability measure which scales on cylinder sets, and therefore $H^{s}\left\lfloor E=H^{s}(E) \mu\right.$. Here $H^{s}\left\lfloor E\right.$ means the restriction of the measure $H^{s}$ to the set $E, H^{s}\left\lfloor E(A):=H^{s}(E \cap A)\right.$. We shall keep this notation for the restriction of a measure. It is easy to see that the open set condition implies $H^{s}\left(E_{i} \cap E_{j}\right)=0$ for any pair $i, j \in M$ with $i \neq j$, and from this it follows that $H^{s}\left(E_{\mathbf{i}} \cap E_{\mathbf{j}}\right)=0$ for $\mathbf{i}, \mathbf{j} \in \mathcal{M}$ such that $\mathbf{i} \wedge \mathbf{j} \notin\{\mathbf{i}, \mathbf{j}\}$. The same is true for any measure multiple of $H^{s}$ and in particular for $\mu$.

\subsection{Densities and measures on a self-similar set}

Next, we recall the definition of Hausdorff type measures obtained by means of a Carathéodory' s process. In what follows for $A \subset \mathrm{R}^{n},|A|$ stands for the diameter of $A$. 
Let $\theta$ be a continuous, increasing function such that $\lim _{x \rightarrow 0^{+}} \theta(x)=0$ and

$$
\limsup _{x \rightarrow 0} \frac{\theta(2 r)}{\theta(r)}<+\infty
$$

Let $\mathscr{C}$ be a family of closed subsets of $\mathrm{R}^{n}$ such that for any $\delta>0$ there exists some $F \in \mathscr{C}$ with $\theta(|F|) \leq \delta$ and $\mathscr{C}$ provides $\delta$-coverings of every set $E \subset \mathrm{R}^{n}$, that is, countable coverings $\left\{F_{k}\right\}$ of $E$ with sets $F_{k} \in \mathscr{C}$ and $\left|F_{k}\right|<\delta$ for all $k$.

For $0<\delta \leq \infty$ and $A \subset \mathrm{R}^{n}$

$$
H_{\mathscr{C}}^{\theta}(A)=\lim _{\delta \rightarrow 0} H_{\mathscr{C}, \delta}(A) \quad \text { where } \quad H_{\mathscr{C}, \delta}(A)=\inf \left\{\sum_{i=1}^{\infty} \theta\left(\left|F_{i}\right|\right)\right\} \text {, }
$$

where the infimum is taken over all countable coverings $\left\{F_{i}\right\}$ of $A$ by subsets of $\mathscr{C}$ with diameter smaller than $\delta$ (see [18] or [30] for details). If we take $\mathscr{C}$ as any of the classes of open subsets, closed subsets or convex subsets of $\mathbf{R}^{n}$ and $\theta: \mathbf{R}^{+} \rightarrow \mathbf{R}^{+}$is a non-decreasing function with $\theta(0)=0$, we get the Hausdorff $\theta$ measure $H^{\theta}$. By taking $\mathscr{C}$ as the class of Euclidean balls of $\mathrm{R}^{n}$, we get the Hausdorff spherical $\theta$ measure $H_{\text {shp }}^{\theta}$. When we choose $\mathscr{C}$ as the class of Euclidean balls centered in $A$, we get the Hausdorff centered $\theta$ premeasure (see Definition 1).

The packing measure is defined in a similar way by replacing coverings with packings. For completeness we recall the definition here.

For $A \subset \mathrm{R}^{n}$ we define

$$
P_{0}^{\theta} A=\lim _{\delta \rightarrow 0} \sup \left\{\sum_{i=1}^{\infty} \theta\left(\left|B_{i}\right|\right)\right\}
$$

where the supremum is taken over all $\delta$-packings of $A$, i.e., countable collections $\left\{B_{i}\right\}$ of disjointed balls centered at $A$ and with diameter smaller than $\delta$. As mentioned in the introduction, the problem that arises when changing optimal coverings by maximal packings is that the limit in (6) fails to be a measure, since $P_{0}^{\theta}$ is subadditive and monotone, but not $\sigma$-subadditive [30].

We define the $\theta$-packing measure as

$$
P^{\theta} A=\inf \left\{\sum_{i=1}^{\infty} P_{0}^{\theta}\left(F_{i}\right)\right\}
$$

where the infimum is taken over all countable coverings $\left\{F_{i}\right\}$ of $A$ by closed sets.

If $\theta(t)=(2 t)^{s}$, we obtain the classical $s$-dimensional Hausdorff, spherical, centered and packing measures, denoted by $H^{s}, H_{s h p}^{s}, C^{s}$ and $P^{s}$, respectively. It is clear that $H^{s} \leq H_{s h p}^{s} \leq 2^{s} H^{s}, C_{0}^{s} \leq 2^{s} H_{s p h}^{s}$ and that $H^{s} \leq C^{s} \leq 2^{s} H^{s}$. 
In [30, Theorem 1, p. 16] Tricot notes the following relations between the Hausdorff centered measure and the upper densities.

For any positive finite Borel measure $v$ in $\mathrm{R}^{n}$, let $\bar{d}_{\nu}^{\theta}(x)=\limsup _{r \rightarrow 0} \frac{v(B(x, r))}{\theta(2 r)}$ be the upper $\theta$-density of $v$ at a point $x \in \mathrm{R}^{n}$. Then

$$
C^{\theta}(E) \inf _{x \in E} \bar{d}_{v}^{\theta}(x) \leq v(E)
$$

and

$$
v(E) \leq C^{\theta}(E) \sup _{x \in E} \bar{d}_{v}^{\theta}(x),
$$

provided $C^{\theta}(E)<\infty$. If we replace $\bar{d}_{v}^{\theta}(x)$ with $\underline{d}_{\nu}^{\theta}(x)$ in (8) and (9) we obtain analogous expressions for the packing measure (see [30]). Notice that Theorem 5 and 10 state that for self-similar sets we can get the Hausdorff centered measure as a minimum (infimum) of the inverse density functions $f_{s}(x, r)=\left(\frac{v(B(x, r))}{\theta(2 r)}\right)^{-1}$.

Observe that for classical gauge functions satisfying (5) we have that $H^{\theta}(E) \leq C^{\theta}(E) \leq\left(\limsup _{t \rightarrow 0} \frac{\theta(2 t)}{\theta(t)}\right) H^{\theta}(E)$ and therefore

$$
\begin{aligned}
H^{\theta}(E)=0 & \Longleftrightarrow C^{\theta}(E)=0 \\
H^{\theta}(E)<\infty & \Longleftrightarrow C^{\theta}(E)<\infty .
\end{aligned}
$$

When we restrict our attention to subsets of $E$, it is easy to see that, if two Borel measures $\mu_{1}$ and $\mu_{2}$ satisfy the scaling property for cylinder sets (i.e., if $\mu_{i}\left(E_{\mathbf{j}}\right)=r_{\mathbf{j}}^{s} \mu_{i}(E), i=1,2, \mathbf{j} \in \mathscr{M}$, and $\left.0<\mu_{2}(E)<\infty\right)$, then $\mu_{1}=\lambda \mu_{2}$ with $\lambda=\frac{\mu_{1}(E)}{\mu_{2}(E)}$. The measures $H^{s}, H_{s p h}^{s}, P^{s}$ and $C^{s}$ defined above are scaling under similitudes and therefore they are, on subsets of $E$, multiples $\lambda \mu$ of the Hausdorff normalized measure $\mu$. The constant $\lambda$ equals to the corresponding measure of the set $E$.

\section{Main results for $C^{s}$}

The next lemma states the self-similar tiling principle which provides the main tool for the results in this paper. From now on $E$ will be a self-similar set in $\mathrm{R}^{n}$ satisfying the OSC.

In this section we will refer a measure $v$ as scaling if it is scaling under similitudes $f_{i}, i \in M$. Observe that all the scaling measures (see (4)) that appear in this note are scaling under similitudes $f_{i}, i \in M$. This is due to the invariance properties of these measures.

Lemma 3.2 (Lemma 4 in [23]). Let $v$ be a scaling measure and $\mu$ be the natural probability measure on $E$. Let $F \subset E$ be a closed set such that 
$\mu F=c>0$ and let $U$ be an open set. Then there exists a subset of indices $\mathscr{J} \subset \mathcal{M}$ such that

$$
\mathscr{J} F \subset U \quad \text { and } \quad \nu U=\nu \mathscr{J} F=\frac{\mu U}{\mu F} \nu F .
$$

Furthermore, $\sum_{\mathbf{i} \in \mathscr{J}} r_{\mathbf{i}}^{s}=\frac{\mu U}{\mu F}$ holds, and given any $\delta>0$, we may require that $\left|E_{\mathbf{i}}\right|<\delta$ for all $\mathbf{i} \in \mathscr{J}$.

If $U$ and $F$ are as in Lemma 3.2 we write $\mathscr{J} \in U: F$ and say that $\mathscr{J}$ is a tiling of $U$ by $F$. Moreover, if we require that $\left|E_{\mathbf{i}}\right|<\delta$ for all $\mathbf{i} \in \mathscr{J}$, we call $\mathscr{J}$ a $\delta$-tiling of $U$ by $F$.

Recall that, given any open set $U \subset E$, there exists a set of indices $\mathscr{I} \subset \mathcal{M}$ such that $U=\cup_{\mathbf{i} \in \mathscr{I}} E_{\mathbf{i}}$. By deleting the redundant cylinders, we may select $\mathscr{I}$ in such a way that the collection of cylinders $\left\{E_{\mathbf{i}}\right\}_{\mathbf{i} \in \mathscr{I}}$ is almost disjoint, i.e., $v\left(E_{\mathbf{i}} \cap E_{\mathbf{j}}\right)=0$ for all $\mathbf{i}, \mathbf{j} \in \mathscr{I}, \mathbf{i} \neq \mathbf{j}$ and for any scaling measure $v$.

In [10] it is proved that for any compact set $K \subset \mathrm{R}^{n}$ with finite packing measure, the extra step in the definition of the packing measure is not needed as $P^{s} K=P_{0}^{s} K$. Next theorem shows that the same result holds for the Hausdorff centered measure in the self-similar setting. However, Example 4 shows that the Hausdorff centered measure does not behave as the packing measure, since there is no equivalent result outside the self-similar setting for $C^{s}$.

THEOREM 3. Let $A$ be either a closed or an open subset of $E$. Then $C_{0}^{s} A=$ $C^{s} A$.

Proof. First we show that it is enough to extend the result of Lemma 3.2 to the premeasure $C_{0}^{s}$. Let $U, F \subset E$ be an open and a closed set with $\mu F>0$, respectively. Suppose that

$$
C_{0}^{s} U=\frac{\mu U}{\mu F} C_{0}^{s} F
$$

and let $A \subset E$ be closed. Now, observe that if we apply (11) first with $E \backslash A$ as open set, and then with $E$ as an open set, we obtain $C_{0}^{s}(E \backslash A)=\frac{\mu(E \backslash A)}{\mu F} C_{0}^{s} F$ and $C_{0}^{s} E=\frac{\mu E}{\mu F} C_{0}^{s} F$. Using these equalities and the subadditiviness of $C_{0}^{s}$, we get

$$
C_{0}^{s} A \geq C_{0}^{s} E-C_{0}^{s}(E \backslash A)=\frac{\mu A}{\mu F} C_{0}^{s} F .
$$

In particular, if $F \subset A$, (12) implies that

$$
C_{0}^{s} A \geq \frac{\mu A}{\mu F} C_{0}^{s} F \geq C_{0}^{s} F .
$$


This also holds for closed subsets $F \subset A$ with $\mu F=0$ since then $C_{0}^{s} F=0$. Thus, by definition of $C^{s}$, we get $C^{s}(A) \leq C_{0}^{s} A$, and the opposite inequality is trivial. This proves the lemma for closed subsets of $E$.

If $A$ is an open subset of $E$, then by (11) and Lemma 3.2 applied to $C^{s}$,

$$
C_{0}^{s} A=\mu A C_{0}^{s} E=\mu A C^{s} E=C^{s} A .
$$

Therefore, it is enough to show that (11) holds. To do so, we consider first the case when $F \subset \mathcal{O}$ and then we extend (11) to general closed sets. We start by repeating the construction of Lemma 4 in [23] to get a $\delta$-tiling $\mathscr{J} \in U: F$ from the $\delta$-tilings $\mathscr{I} \in U: E$ and $\mathscr{K} \in(E \backslash F): E$. For $k \in \mathrm{N}^{+}$, set $\mathscr{J}_{k+1}=\mathscr{J}_{k} \vee \mathscr{K}$ with $\mathscr{J}_{0}=\mathscr{I}$. Noting that

$$
\begin{aligned}
U=\mathscr{J}_{0} E & =\mathscr{J}_{0} F \cup \mathscr{J}_{0}(E \backslash F)=\mathscr{I}_{0} F \cup\left(\mathscr{J}_{0} \vee \mathscr{K}\right) E \\
& =\mathscr{J}_{0} F \cup \mathscr{J}_{1} F \cup\left(\mathscr{J}_{1} \vee \mathscr{K}\right) E=\ldots=\left(\bigcup_{p=0}^{k} \mathscr{J}_{p} F\right) \cup \mathscr{J}_{k+1} E
\end{aligned}
$$

and setting $\mathscr{J}=\bigcup_{k=0}^{\infty} \mathscr{J}_{k}$, we obtain a $\delta$-tiling of $U$ by $F$ such that $\mathscr{J} F \subset U$ and

$$
v(U)=v(\mathscr{J} F)=\sum_{\mathbf{i} \in \mathscr{J}} r_{\mathbf{i}}^{s} v F=\sum_{k=0}^{\infty} \mu U(1-v F)^{k} \nu F=\frac{\mu U}{\mu F} \nu F
$$

for any scaling measure $v$ and any closed set $F \subset E$ with $\mu F>0$ (see the proof of Lemma 4 in [23]). Observe that the first equality in (14) shows that $\mathscr{J} F$ is dense in $U$.

Let $\varepsilon>0$. The convergence of the sum in (14) implies that, for $p$ big enough and $v=C^{s}$,

$$
\sum_{\mathbf{i} \in \mathscr{J}} r_{\mathbf{i}}^{s} C_{0}^{s} F \leq \sum_{\mathbf{i} \in \mathscr{T}_{p}} r_{\mathbf{i}}^{s} C_{0}^{s} F+\varepsilon
$$

where $\mathscr{T}_{p}:=\bigcup_{k=0}^{p} \mathscr{J}_{k}$.

Note that from the hypothesis $F \subset \mathscr{O}$ and $\mu F>0$ it follows that $F_{\mathbf{i}} \cap F_{\mathbf{j}}=\emptyset$ for any $\mathbf{i}, \mathbf{j} \in \mathscr{T}_{p}$ with $\mathbf{i} \neq \mathbf{j}$. To see this, take $t>m$ and let $\mathbf{i}=\left(i_{1}, \ldots, i_{m}\right)$ and $\mathbf{j}=\left(j_{1}, \ldots, j_{t}\right) \in \mathscr{T}_{p}$. If $\mathbf{i} \wedge \mathbf{j}=\mathbf{i}_{0}$, then, as $F \subset \mathscr{O}, F_{\mathbf{i}} \cap F_{\mathbf{j}} \subset \mathscr{O}_{i_{1}} \cap \mathscr{O}_{j_{1}}=\emptyset$. If $\mathbf{i} \wedge \mathbf{j}=\left.\mathbf{i}\right|_{q}$ with $q<m$, then $F_{\mathbf{i}} \cap F_{\mathbf{j}} \subset f_{\mathbf{i}_{q}}\left(\mathscr{O}_{i_{q+1}} \cap \mathscr{O}_{j_{q+1}}\right)=\emptyset$. If $m=q$, $\mathbf{i} \in \mathscr{J}_{l}$ and $\mathbf{j} \in \mathscr{J}_{k}$ with $l \neq k$, then $\mathbf{i} \wedge \mathbf{j}=\mathbf{i} \in \mathscr{J}_{l}$ and $\overline{\mathbf{j}}=:\left(j_{m+1}, \ldots, j_{t}\right) \in$ $\mathscr{K} \vee \ldots \vee \mathscr{K}$, therefore $F_{\overline{\mathbf{j}}} \subset E \backslash F$. This implies that $F_{\mathbf{i}} \cap F_{\mathbf{j}}=f_{\mathbf{i}}\left(F \cap F_{\overline{\mathbf{j}}}\right)=\emptyset$. Finally, the case $m=q$ with $l=k$ is not possible by construction since this 
would imply the existence of sequences $\mathbf{i}, \mathbf{j}$ either in $\mathscr{I}$ or in $\mathscr{K}$ such that $\mathbf{i} \wedge \mathbf{j}=\mathbf{i}$ and this is not possible because $\mathscr{I}$ and $\mathscr{K}$ are almost disjoint tilings.

Thus, for a sufficiently small $\delta$ any covering of $\mathscr{J} F$ by balls centered in $\mathscr{J} F$ and with diameter smaller that $\delta$ provides admissible disjointed coverings of all the copies $F_{\mathbf{i}}, \mathbf{i} \in \mathscr{T}_{p}$. Hence, $C_{0}^{s} \mathscr{J} F \geq \sum_{\mathbf{i} \in \mathscr{T}_{p}} C_{0}^{s} F_{\mathbf{i}}$. Now, as $C_{0}^{s}$ is a scaling premeasure and by (15) we have

$$
C_{0}^{s} \mathscr{J} F \geq \sum_{\mathbf{i} \in \mathscr{T}_{p}} C_{0}^{s} F_{\mathbf{i}}=\sum_{\mathbf{i} \in \mathscr{T}_{p}} r_{\mathbf{i}}^{s} C_{0}^{s} F \geq \sum_{\mathbf{i} \in \mathscr{J}} r_{\mathbf{i}}^{s} C_{0}^{s} F-\varepsilon .
$$

Letting $\varepsilon$ go to zero we get $C_{0}^{s} \mathscr{J} F \geq \sum_{\mathbf{i} \in \mathscr{F}} r_{\mathbf{i}}^{s} C_{0}^{s} F$. The opposite inequality holds because $C_{0}^{s}$ is $\sigma$-subadditive. Hence,

$$
C_{0}^{s} \mathscr{J} F=\sum_{\mathbf{i} \in \mathscr{J}} C_{0}^{s} F_{\mathbf{i}}
$$

Now, using that $\mathscr{J} F$ is dense in $U,(16)$ and (14) we obtain that

$$
C_{0}^{s} U \geq C_{0}^{s} \mathscr{J} F=\sum_{\mathbf{i} \in \mathscr{J}} r_{\mathbf{i}}^{s} C_{0}^{s} F=\frac{\mu U}{\mu F} C_{0}^{s} F .
$$

Moreover, since $C_{0}^{s}$ is $\sigma$-subadditive it turns out that

$$
C_{0}^{s} U \leq C_{0}^{s} \mathscr{J} F+C_{0}^{s}(U \backslash \mathscr{J} F)=\frac{\mu U}{\mu F} C_{0}^{s} F .
$$

The last equality holds because $C_{0}^{s} \leq P^{s}$ (see [30, Theorem 1]) and $P^{s}$ is a scaling measure verifying (14) and therefore $C_{0}^{s}(U \backslash \mathscr{J} F) \leq P^{s}(U \backslash \mathscr{J} F)=0$. Thus, (17) together with (18) conclude the case $F \subset \mathcal{O}$.

For the general case, consider a sequence $\left\{F_{k}\right\}$ of closed subsets of $E$ with $F_{k} \subset F \cap \mathscr{O}$ for all $k \in \mathrm{N}^{+}$and $\bigcup_{k=1}^{\infty} F_{k}=F \cap \mathscr{O}$. Let $U \subset E$ be an open set. Then, by subadditiveness,

$$
C_{0}^{s} U=\frac{\mu U}{\mu F_{k}} C_{0}^{s} F_{k} \geq \frac{\mu U}{\mu F_{k}}\left(C_{0}^{s} F-C_{0}^{s}\left((F \cap \mathscr{O}) \backslash F_{k}\right)-C_{0}^{s}(F \backslash \mathscr{O})\right)
$$

for all $k \in \mathrm{N}^{+}$. As $C_{0}^{s}(F \backslash \mathcal{O})=0$ and $C_{0}^{s}\left((F \cap \mathcal{O}) \backslash F_{k}\right)$ tends to zero as $k$ tends to infinity, we get that $C_{0}^{s} U \geq \frac{\mu U}{\mu F} C_{0}^{s} F$. As $C_{0}^{s}$ is subadditive then, the chain (18) also holds in this case if we replace the last equality with the inequality $\leq$. Therefore $C_{0}^{s} U \leq \frac{\mu U}{\mu F} C_{0}^{s} F$ and (11) is proved.

In [30] Tricot gave an example to show the lack of monotonicity for the premeasure $C_{0}^{s}$. The same example can be used to show that Theorem 3 cannot be extended to the non-selfsimilar case. 
Example 4 ([30, p. 18]). Let $S$ be the self-similar set obtained as the invariant set of three similarities $\left\{f_{1}, f_{2}, f_{3}\right\}$ of contraction ratio $r_{1}=r_{2}=$ $r_{3}=1 / 3$, each sending the equilateral triangle into three smaller triangles (see Figure 1).

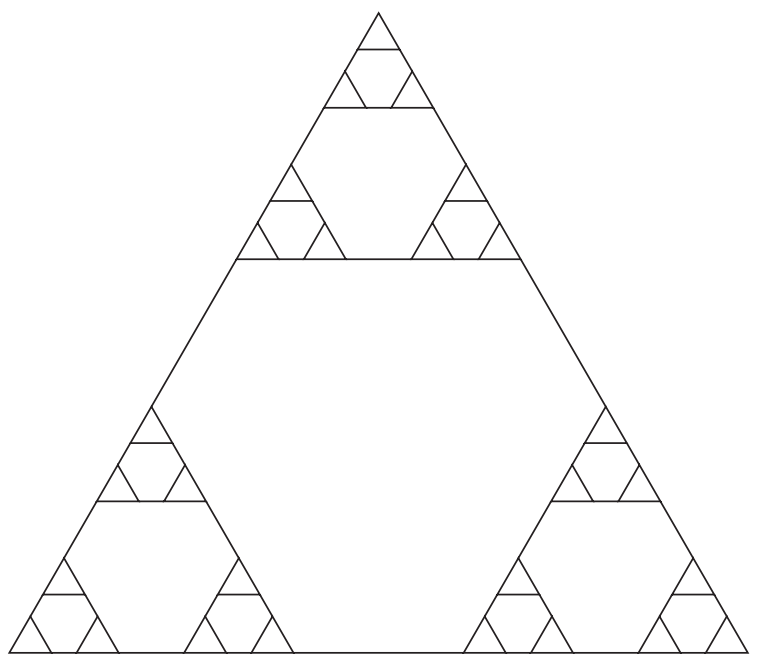

FIGURE 1. $S$ is the invariant set for the similarities $f_{1}(x, y)=\frac{1}{3}(x, y)$, $f_{2}(x, y)=\frac{1}{3}(x, y)+\left(\frac{2}{3}, 0\right), f_{3}(x, y)=\frac{1}{3}(x, y)+\left(\frac{1}{3}, \frac{1}{\sqrt{3}}\right)$.

This invariant set is a Sierpinski triangle of dimension $s=1$. Let $N$ be the set of all centers of the hexagons of each generation. In [30] it is shown that

$$
C_{0}(S \cup N)<C_{0}(S) .
$$

Therefore, if we let $F=S \cup N$ and we apply Theorem 3 to $S$, we get

$$
C^{s} F=C^{s} S=C_{0}^{s} S>C_{0}^{s} F .
$$

Observe that the first equality in (19) holds trivially since $N$, as countable, is a null set for $C^{1}$.

In [23], the self-similar tiling principle has been used to give the exact value of Hausdorff-type scaling measures on $E$ by means of the minimal inverse density in the class of covering sets. In [14] it is proved that under strong separation condition (SSC) these optimal values are attained. We say that the self-similar set $E$ satisfies the strong separation condition (SSC) if the union $E=\bigcup_{i=1}^{n} f_{i}(E)$ is disjoint. The ideas in [23] and [14] together with Theorem 3 gives readily the value of the Hausdorff centered measure. 
THEOREM 5. Let $E$ be the invariant set of the system $\Psi$ satisfying the open set condition with $\operatorname{dim}_{H} E=s$ and $|E|=R$ and let $\mu$ be the Hausdorff normalized measure on E. Then

$$
C^{s} E=\inf \left\{\frac{(2 r)^{s}}{\mu(B(x, r))}: x \in E, r>0\right\}=: D_{C}^{-1}
$$

Moreover, if $\Psi$ satisfies the SSC then

$$
C^{s} E=\min \left\{\frac{(2 r)^{s}}{\mu(B(x, r))}: x \in E \text { and } c \leq r \leq R\right\}
$$

where $c:=\min _{i, j \in M, i \neq j}\left(f_{i} E, f_{j} E\right)$ and $R:=|E|$.

REMARK 6. We denote by $B(x, r)$ the closed ball centered at $x \in \mathrm{R}^{n}$ and with radius $r>0$. One difficulty that we need to overcome stems from the fact that the balls in $E$ as a metric subspace of $\mathrm{R}^{n}$,

$$
B_{E}(x, r):=B(x, r) \cap E=\{y \in E: d(x, y) \leq r\},
$$

are not invariant under similitudes $f_{i}, i \in M$. Here $d$ is the euclidean distance. The inclusion $f_{i} B_{E}(x, r) \subset B_{E}\left(x_{i}, r_{i} r\right)$ always holds, but it may be strict because $f_{i}$ restricted to $E$ is not a surjective mapping. In [14, Remark 3.2] it is noted that

$$
B(x, r) \cap E=f_{i}\left(B\left(f_{i}^{-1}(x), r / r_{i}\right) \cap E\right)
$$

whenever $B(x, r) \cap E \subset E_{i}$. Hence for any pair $(x, r)$ satisfying that $B(x, r) \cap$ $E \subset E_{i}$, we have that

$$
\frac{(2 r)^{s}}{\mu B(x, r)}=\frac{\left(2 r_{1}\right)^{s}}{\mu B\left(x, r_{1}\right)}
$$

where $r_{1}=\frac{r}{r_{i}}$ and $x_{1}=f_{i}^{-1}\left(x_{0}\right)$ (see Theorem 3.3 in [14]).

Proof. First we are going to show the inequality $C^{s} E \leq D_{C}^{-1}$. Let $\varepsilon>0$ and $B=: B(x, r)$ be a closed ball centered in $E$ with radius $r>0$ and such that $\frac{|B|^{s}}{\mu(B)} \leq D_{C}^{-1}+\varepsilon$. Note that if, for any such $B$, we can find a subset $K \subset E$ satisfying that $C_{0}^{s} E \leq C_{0}^{s} K \leq \frac{|B|^{s}}{\mu(B)}$, then Theorem 3 implies that $C^{s} E=C_{0}^{s} E \leq C_{0}^{s} K \leq D_{C}^{-1}+\varepsilon$ and the inequality is proved.

Let $\left\{\delta_{k}\right\}$ be a sequence of positive numbers tending to zero. For $k \in \mathrm{N}$ let $\mathscr{J}_{k} \in E:(B \cap E)$ be a $\delta_{k}$-tiling of $E$ by $B \cap E$. We define $K=: \bigcap_{k} \mathscr{J}_{k} B$. 
Let $k \in \mathrm{N}$. Observe that $\mu B>0$ and that $C^{s}$ is a scaling measure. Hence we can apply Lemma 3.2 to $C^{s}\left\lfloor E\right.$, giving that $C^{s}\left(E \backslash \mathscr{J}_{k} B\right)=C_{0}^{s}\left(E \backslash \mathscr{J}_{k} B\right)=0$. Moreover, it follows from Lemma 3.2 that

$$
\sum_{\mathbf{i} \in \mathscr{f}_{k}} r_{\mathbf{i}}^{s}=\frac{\mu E}{\mu B}=\frac{1}{\mu B} .
$$

Hence

$$
\sum_{\mathbf{i} \in \mathscr{F}_{k}}\left|B_{\mathbf{i}}\right|^{s}=\sum_{\mathbf{i} \in \mathscr{C}_{k}} r_{\mathbf{i}}^{s}|B|^{s}=\frac{|B|^{s}}{\mu B} .
$$

Noticing that, for any $k \in \mathrm{N}, \mathscr{J}_{k} B$ provides a $\delta_{k}$-covering of $E$, we obtain

$$
C_{0}^{s}(K) \leq \lim _{k \rightarrow \infty}\left\{\sum_{\mathbf{i} \in \mathscr{F}_{k}}\left|B_{\mathbf{i}}\right|^{s}\right\}=\frac{|B|^{s}}{\mu B} .
$$

Recall that $C_{0}^{s}$ is not a measure since it is not monotone. However, as it is $\sigma$-subadditive and we know that $C_{0}^{s}\left(E \backslash \mathscr{J}_{k} B\right)=0$, we have

$$
\begin{aligned}
C^{s}(E) & =C_{0}^{s}(E) \leq C_{0}^{s}(E \backslash K)+C_{0}^{s}(K)=C_{0}^{s}\left(E \backslash \bigcap_{k=1}^{\infty} \mathscr{J}_{k} B\right)+C_{0}^{s}(K) \\
& =C_{0}^{s}\left(\bigcup_{k=1}^{\infty}\left(E \backslash \mathscr{J}_{k} B\right)\right)+C_{0}^{s}(K) \leq \sum_{k=1}^{\infty} C_{0}^{s}\left(E \backslash \mathscr{J}_{k} B\right)+C_{0}^{s}(K) \\
& =C_{0}^{s}(K) .
\end{aligned}
$$

So the first inequality is proved.

In order to show the reverse inequality, take any countable covering $\left\{W_{k}\right\}$ of $E$ by closed balls centered at $E$. Let $k_{i}, i=1,2,3, \ldots$ be the sequence of subindices corresponding to the sets in $\left\{W_{k}\right\}$ with positive $\mu$ measure. Then

$$
\begin{aligned}
\sum_{k=1}^{\infty}\left|W_{k}\right|^{s} & \geq \sum_{i=1}^{\infty}\left|W_{k_{i}}\right|^{s}=\sum_{i=1}^{\infty} \frac{\left|W_{k_{i}}\right|^{s}}{\mu\left(W_{k_{i}}\right)} \mu\left(W_{k_{i}}\right) \geq D_{C}^{-1} \sum_{i=1}^{\infty} \mu\left(W_{k_{i}}\right) \\
& \geq D_{C}^{-1} \mu\left(\bigcup_{i=1}^{\infty} W_{k_{i}}\right)=D_{C}^{-1} .
\end{aligned}
$$

Therefore, $C^{s} E \geq C_{0}^{s} E \geq D_{C}^{-1}$ and (20) is proved.

The proof of (21) follows from (23) as in [14, Theorem 3.3] where an analogous result is proved for the Hausdorff, packing and Hausdorff spherical 
measures. Denote by

$$
c:=\min _{i, j \in M, i \neq j}\left(f_{i} E, f_{j} E\right) .
$$

By definition of $c$, it is clear that for any $(x, r) \in E \times(0, c)$ there exists $i \in M$ such that $B(x, r) \subset E_{i}$ and thus, we can apply (23) to get $\frac{(2 r)^{s}}{\mu B(x, r)}=\frac{\left(2 r_{1}\right)^{s}}{\mu B\left(x, r_{1}\right)}$ where $r_{1}=\frac{r}{r_{i}}$ and $x_{1}=f_{i}^{-1}\left(x_{0}\right)$. We want to show that

$$
\inf _{E \times(0, \infty)} \frac{(2 r)^{s}}{\mu(B(x, r))}=\inf _{E \times[c, R]} \frac{(2 r)^{s}}{\mu(B(x, r))} .
$$

Suppose that there exists $\left(x_{0}, r_{0}\right) \in E \times(0, c)$ satisfying that

$$
\frac{\left(2 r_{0}\right)^{s}}{\mu\left(B\left(x_{0}, r_{0}\right)\right)}<\inf _{E \times[c, R]} \frac{(2 r)^{s}}{\mu(B(x, r))} .
$$

Let $\mathbf{i} \in M^{\infty}$ be the address of $x_{0}$ and $k \in \mathbf{N}$. Let $x_{k}=f_{\mathbf{i}(k)}^{-1}\left(x_{0}\right)$ and $r_{k}=\frac{r_{0}}{r_{\mathbf{i}(k)}}$. Then, for some $k_{0} \in \mathrm{N}$, we have $r_{k_{0}} \geq c, r_{k_{0}-1} \leq c$ and $x_{k_{0}} \in E$. Thus, we can apply (23) repeatedly to get that

$$
\frac{\left(2 r_{0}\right)^{s}}{\mu\left(B\left(x_{0}, r_{0}\right)\right)}=\frac{\left(2 r_{k_{0}}\right)^{s}}{\mu\left(B\left(x_{k_{0}}, r_{k_{0}}\right)\right)} .
$$

So $r_{0} \geq c$.

Finally, if $r_{0}>R$ then $\frac{\left(2 r_{0}\right)^{s}}{\mu\left(B\left(x_{0}, r_{0}\right)\right)}=\left(2 r_{0}\right)^{s}>(2 R)^{s}$. So then, $r_{0} \in[c, R]$ and the theorem is proved.

\section{General Hausdorff centered measure $C^{\theta}$}

In this section we are going to extend Theorem 3 and Theorem 5 to general Hausdorff centered measures $C^{\theta}$ associated to suitable gauge functions $\theta$. The key property of $\theta$ is that the corresponding $C^{\theta}$ scales. In the next lemma we characterize the gauge functions with this property.

Lemma 7. Let $n$ be a positive integer. Let $\theta$ be a continuous increasing function defined on $\mathbf{R}^{+}$such that $\theta(0)=0$ and $\theta(t) / t^{n}$ is a decreasing function of $t$. Then

$$
\lim _{t \rightarrow 0} \frac{\theta(c t)}{\theta(t)}=c^{s} \quad \forall c>0 \Leftrightarrow C^{\theta}(c A)=c^{s} C^{\theta}(A) \quad \forall c>0, A \subset \mathrm{R}^{n} .
$$

The proof of this lemma is inspired by [1, Theorems 2 and 3]. There, Csörnyei and Mauldin, completely characterized the gauge functions $\theta$ for which the measures $H^{\theta}$ and $P^{\theta}$ scale with exponent $s$. They proved that under 
the conditions of Lemma 7 the left part in (26) is equivalent to the following statements

(a) $H^{\theta}(c A)=c^{s} H^{\theta}(A) \quad \forall c>0, A \subset \mathrm{R}^{n}$

(b) $P^{\theta}(c A)=c^{s} P^{\theta}(A) \quad \forall c>0, A \subset \mathrm{R}^{n}$.

In other words, the packing and Hausdorff measures $H^{\theta}$ and $P^{\theta}$ scale if and only if $\theta(t)=t^{s} L(t)$, where $L$ is a slowly varying function in the sense of Karamata. We say that $L: \mathrm{R} \rightarrow \mathrm{R}$ is a slowly varying function if for every $c>0$ it holds that

$$
\lim _{x \rightarrow \infty} \frac{L(c x)}{L(x)}=1 .
$$

More precisely, (a) and (b) follow from the following theorem.

THEOREM 8 (Csörnyei and Mauldin [1], Theorem 2). For every function $f: \mathbf{R} \rightarrow \mathbf{R}^{+}$, the following are equivalent:

(i) $H^{\theta}(c A) \leq f(c) H^{\theta}(A) \quad \forall c>0, A \subset \mathrm{R}^{n}$;

(ii) $P^{\theta}(c A) \leq f(c) P^{\theta}(A) \quad \forall c>0, A \subset \mathrm{R}^{n}$;

(iii) $\lim \sup _{t \rightarrow 0} \frac{\theta(c t)}{\theta(t)} \leq f(c) \quad \forall c>0$.

Analogously, for every function $g: \mathrm{R} \rightarrow \mathrm{R}^{+}$, the following are equivalent:

(iv) $H^{\theta}(c A) \geq g(c) H^{\theta}(A) \quad \forall c>0, A \subset \mathrm{R}^{n}$;

(v) $P^{\theta}(c A) \geq g(c) P^{\theta}(A) \quad \forall c>0, A \subset \mathrm{R}^{n}$;

(vi) $\liminf _{t \rightarrow 0} \frac{\theta(c t)}{\theta(t)} \geq g(c) \quad \forall c>0$.

The proof of Lemma 7 is given at the end of the section. First, we are going to see that Theorem 3 extends precisely to those gauge functions described in Lemma 7.

THEOREM 9. Let $n$ be a positive integer. Let $\theta$ be a continuous increasing function defined on $\mathrm{R}^{+}$, such that $\theta(0)=0$ and $\theta(t) / t^{n}$ is a decreasing function of t verifying that $\lim _{t \rightarrow 0} \frac{\theta(c t)}{\theta(t)}=c^{s} \forall c>0$. Let $F \subset E$ be a subset of $E$ either closed or open. Then $C_{0}^{\theta} F=C^{\theta} F$.

Proof. Replace $C^{s}$ with $C^{\theta}$ and $C_{0}^{s}$ with $C_{0}^{\theta}$, respectively, in the proof of Theorem 3. Observe that the only property of $C^{s}$ and $C_{0}^{s}$ used in the proof of this theorem is that these measures are scaling, and this is provided by Lemma 7. Note that, to prove (26), we have got first the results for $C_{0}^{\theta}$ and then for $C^{\theta}$ by taking the supremum. So, in particular, $C_{0}^{\theta}$ scales.

In contrast with Theorem 9, for the extension of Theorem 5 to general measures, the scaling properties of $C^{\theta}$ and $C_{0}^{\theta}$ are not enough. Here the application of the self-similar tiling principle requires some additional conditions in the 
gauge function. In particular $L(t)$ must be, in addition to slowly varying, non decreasing.

THEOREM 10. Let $E$ be the invariant set of the system $\Psi$ satisfying the open set condition with $\operatorname{dim}_{H} E=s$ and $|E|=R, \mu$ be the Hausdorff normalized measure on $E$ and $\theta(t)=t^{s} L(t)$ where $L(t)$ is a non decreasing slowly varying function. Then

$$
C^{\theta} E=\inf \left\{\frac{\theta(2 r)}{\mu(B(x, r))}: x \in E, r>0\right\}=: D_{C}^{-1}
$$

Moreover, if $\Psi$ satisfies the SSC then

$$
C^{\theta} E=\min \left\{\frac{\theta(2 r)}{\mu(B(x, r))}: x \in E \text { and } c \leq r \leq R\right\}
$$

where $c:=\min _{i, j \in M, i \neq j}\left(f_{i} E, f_{j} E\right)$ and $R:=|E|$.

Proof. To show (27), replace $C^{s}$ and $C_{0}^{s}$ with $C^{\theta}$ and $C_{0}^{\theta}$, respectively, in the proof of Theorem 5 and apply Theorem 9 instead of Theorem 3. Then observe that, in order to get the inequality $C^{\theta} E \geq D_{C}^{-1}$, the chain (24) should be replaced with

$$
\sum_{\mathbf{i} \in \mathscr{F}_{k}} \theta\left(\left|B_{\mathbf{i}}\right|\right)=\sum_{\mathbf{i} \in \mathscr{\mathscr { F }}_{k}} r_{\mathbf{i}}^{s} \theta(|B|) \frac{L\left(\left|B_{\mathbf{i}}\right|\right)}{L(|B|)} \leq \sum_{\mathbf{i} \in \mathscr{F}_{k}} r_{\mathbf{i}}^{s} \theta(|B|)=\frac{\theta(|B|)}{\mu B} .
$$

Here we are using that $L(t)$ is a non decreasing function. The rest of the proof is the same.

To get (28) note that, whenever $B(x, r) \cap E \subset E_{i}$, (22) implies

$$
\begin{aligned}
\frac{\theta(2 r)}{\mu B(x, r)} & =\frac{H^{s}(E) \theta(2 r)}{H^{s}(E \cap B(x, r))}=\frac{H^{s}(E) \theta(2 r)}{r_{i}^{s} H^{s}\left(E \cap B\left(x_{1}, r_{1}\right)\right)} \\
& =\frac{H^{s}(E) \theta\left(2 r_{1}\right)}{H^{s}\left(E \cap B\left(x_{1}, r_{1}\right)\right)} \frac{L\left(2 r_{1}\right)}{L(2 r)} \geq \frac{\theta\left(2 r_{1}\right)}{\mu B\left(x, r_{1}\right)} .
\end{aligned}
$$

Therefore, the same arguments used in the proof of (21) are valid in this case by replacing the equality (25) with

$$
\frac{\theta\left(2 r_{0}\right)}{\mu\left(B\left(x_{0}, r_{0}\right)\right)} \geq \frac{\theta\left(2 r_{k_{0}}\right)}{\mu\left(B\left(x_{k_{0}}, r_{k_{0}}\right)\right)}
$$

Finally, since $\theta(t)$ is increasing, if $r_{0}>R$ then $\frac{\theta\left(2 r_{0}\right)}{\mu\left(B\left(x_{0}, r_{0}\right)\right)}=\theta\left(2 r_{0}\right)>\theta(2 R)$. 
Now we turn to the proof of Lemma 7. Our aim is to follow Csörnyei and Mauldin's strategy for the Hausdorff centered measure. Thus, we need to show that statement (iii) in Theorem 8 is equivalent to

$$
C^{\theta}(c A) \leq f(c) C^{\theta}(A) \quad \forall c>0, A \subset \mathrm{R}^{n}
$$

and that (vi) is equivalent to

$$
C^{\theta}(c A) \geq g(c) C^{\theta}(A) \quad \forall c>0, A \subset \mathrm{R}^{n} .
$$

A modification of the proof given in [1] works also in this case. Namely, we will need to modify their construction since we need to keep control on the centers of the covering balls. We are going to describe only these changes, referring the reader to [1] for the complete argument.

Proof of Lemma 7. Following [1], we just need to show that (iii) implies (29) and (30) implies (vi). The first part is equivalent to the proof of (iii) implies (i) in [1] by changing the coverings by centered coverings (so we get $\left.C_{0}^{\theta}(c A) \leq f(c) C_{0}^{\theta}(A)\right)$ and then passing to the supremum to obtain (29). The main changes are needed in the proof of "(30) implies (vi)".

Take $c>0$ and choose $z_{1}>z_{2}>\cdots \rightarrow 0$ such that

$$
\lim _{n \rightarrow \infty} \frac{\theta\left(c z_{n}\right)}{\theta\left(z_{n}\right)}=\liminf _{t \rightarrow 0} \frac{\theta(c t)}{\theta(t)}
$$

It is enough to show that for all $\epsilon>0$, there exists a set $K$, a probability measure $\mu$, a positive finite number $M$ and an $\epsilon$-covering of $K$ by balls centered in $K, G_{\epsilon}$, such that

(1) $\mu(K)=1$

(2) $\theta(\operatorname{diam} A) \geq M \mu(A)$ for all $A \subset \mathrm{R}^{n}$

(3) $\sum_{B \in G_{\epsilon}} \theta(\operatorname{diam} B) \rightarrow M$ as $\epsilon \rightarrow 0$

(4) $\operatorname{diam}(B) \in\left\{z_{1}, z_{2}, \ldots\right\}$ for all $B \in G_{\epsilon}$.

To see that we can reduce to this construction, we refer the interested reader to [1]. There is a minor modification needed due to the two step definition of the Hausdorff centered measure, but it can be done by standard arguments.

Thus, we just need to construct $K, \mu$ and $G_{\epsilon}$ verifying (1)-(4). The main difference between the Hausdorff and the Hausdorff centered measure stands on the nature of the coverings, in our case the balls in $G_{\epsilon}$ must be centered in $K$. As $K$ is given by (31) and, for each $\epsilon>0, G_{\epsilon}$ will be given in terms of $\bigcup_{i=1}^{M_{n}} B_{i n}$, then to change the nature of the coverings we need to modify the set $K$. Also note that, since we are just interested in the Euclidean case, we take 
$n$-dimensional cubes instead of the regular bricks considered in by Csörnyei and Mauldin.

In what follows, we will explain the basic steps and changes needed for this construction.

For all $n \in \mathrm{N}$ choose a finite set

$$
C^{n}=\left\{C_{1 n}, \ldots, C_{M_{n} n}\right\}
$$

of cubes of size $x_{n} \in\left\{z_{1}, z_{2}, \ldots\right\}$, such that $\cup C^{n+1} \subset \cup C^{n}$ and there is $\frac{M_{n+1}}{M_{n}}$ cubes of generation $n+1$ inside each $C_{j n}$. Let $B_{j n}$ be the ball of diameter $x_{n}$ such that $B_{j n} \subset C_{j n}$. The diameters $x_{n}$ and the constants $M_{n}$ and $M$ are taken such that $\left\{x_{n}\right\}$ is a subsequence of $\left\{z_{n}\right\}$ (so $x_{n} \rightarrow 0$ ) and $M_{n} \theta\left(x_{n}\right) \rightarrow M$.

Suppose $x_{n}$ and $C^{n}$ has been defined, $x_{n+1}$ and $C^{n+1}$ are defined in the following way. Put a regular grid $N \times N \times \cdots \times N$ onto each of the cubes $C_{j n}$ and choose those small cubes determined by the grid that are inside $B_{j n}$. Observe that if $N$ is an odd number then, one of the chosen cubes must be centered in the center of $B_{j n}$. To get $C^{n+1}$ we need to shrink the chosen cubes. In this way, $C^{n+1}$ is the set of smaller cubes with the same midpoints as the chosen cubes and of size $x_{n+1}$ (in [1] one can see that $x_{n+1}$ must be much smaller than $\frac{x_{n}}{N}$, to be precise, $x_{n+1}=\epsilon_{n} \frac{x_{n}}{N}$, where $\epsilon_{n}$ is "small enough")

The measure $\mu$ is taken as the unique probability measure verifying that $\mu\left(C_{i n}\right)=\frac{1}{M_{n}}$,

$$
K=\bigcap_{n=1}^{\infty} \bigcup_{i=1}^{M_{n}} B_{i n}
$$

and $G_{\epsilon}=\left\{B_{1 n}, B_{2 n}, \ldots, B_{M_{n} n}\right\}$ with $n=n(\epsilon)$ big enough so $\left|B_{i n}\right| \leq \epsilon$ for all $i=1, \ldots, M_{n}$.

As noticed before, since $N$ is an odd number, then there is always a cube in $C^{n+1}$ centered in the center of $B_{j n}$. This, the definition of $G_{\epsilon}$ and (31) ensures that the center of any ball $B_{j n}$ belongs to $K$ and hence $G_{\epsilon}$ is an admissible covering for the Hausdorff centered measure. So then, to get our result, we just need to take $N$ in [1] to be odd. And this extra condition does not change the proof, because the only property of $N$ used in [1] is that it has to be a big number. Hence, $C_{0}^{\theta}(c A) \geq g(c) C_{0}^{\theta}(A)$ for all $c>0$ and $A \subset \mathrm{R}^{n}$ and (30) holds by taking the supremum.

\section{REFERENCES}

1. Csörnyei, M., and Mauldin, R. D., Scaling properties of Hausdorff and packing measures, Math. Ann. 319 (2001), 817-836.

2. Csörnyei, M., An example illustrating $P^{g}(K) \neq P_{0}^{g}(K)$ for compact sets of finite premeasure, Real Anal. Exchange 27 (2001/02), 65-70. 
3. Edgar, G. A., Fine variation and fractal measures, Real Anal. Exchange 20 (1995), 256-280.

4. Edgar, G. A., Integral, Probability and Fractal Measure, Springer, New York 1998.

5. Edgar, G. A., Centered densities and fractal measures, New York J. Math. 13 (2007), 33-87.

6. Falconer, K. J., and Howroyd, J. D., Projection theorems for box and packing dimensions, Math. Proc. Cambridge Philos. Soc. 119 (1996), 287-295.

7. Falconer, K. J. and Järvenpää, M., Packing dimensions of sections of sets, Math. Proc. Cambridge Philos. Soc. 125 (1999), 89-104.

8. Falconer, K. J., Järvenpää, M., and Mattila, P., Examples illustrating the instability of packing dimensions of sections, Real Anal. Exchange 25 (1999/2000), 629-640.

9. Falconer, K. J., and Mattila, P., The packing dimension of projections and sections of measures, Math. Proc. Cambridge Philos. Soc. 119 (1996), 695-713.

10. Feng D., Hua, S., and Wen, Z.-Y., Some relations between packing premeasure and packing measure, Bull. London Math. Soc. 31 (1998), 665-670.

11. Hutchinson, J. E., Fractals and self-similarity, Indiana Univ. Math. J. 30 (1981), 713-747.

12. Järvenpää, M., and Mattila, P., Hausdorff and packing dimensions and sections of measures, Mathematika 45 (1998), 55-77.

13. Järvenpää, E., Järvenpää, M., and Llorente, M., Local dimensions of sliced measures and stability of packing dimensions of sections of sets, Adv. Math. 183 (2004), 127-154.

14. Llorente, M., and Morán, M., Self-similar sets with optimal coverings and packings, J. Math. Anal. Appl. 334 (2007), 1088-1095.

15. Marion, J., Measures de Hausdorff d'ensembles fractales, Ann. Sci. Math. Québec 11 (1987), 111-132.

16. Marstrand, M., Some fundamental geometrical properties of plane sets of fractional dimension, Proc. London Math. Soc. (3) 4 (1954), 257-302.

17. Mattila, P., On the structure of self-similar fractals, Ann. Acad. Sci. Fenn. (A I) 7 (1982), 189-195.

18. Mattila, P., Geometry of Sets and Measures in Euclidean Spaces, Cambridge Studies in Adv. Math. 44, Cambridge Univ. Press, Cambridge 1995.

19. Mattila, P., Hausdorff dimension, orthogonal projections and intersections with planes, Ann. Acad. Sci. Fenn. (A I) 1 (1975), 227-244.

20. Mattila, P., Hausdorff dimension, projections, and the Fouriertransform, Publ. Mat. 48(2004), 3-48.

21. Meifeng, D., and Lixin,T., Exact Hausdorff centered measure of symmetry Cantor sets, Chaos Solitons Fractals 26 (2005), 313-323.

22. Morán, M., Dynamical boundary of a self-similar set, Fund. Math. 160 (1999), 1-14.

23. Morán M., Computability of the Hausdorff and packing measures on self-similar sets and the self-similar tiling principle, Nonlinearity 18 (2005), 559-570.

24. Saint Raymond, X., and Tricot, C., Packing regularity of sets in n-space, Math. Proc. Cambridge Philos. Soc. 103 (1988), 133-145

25. Schechter, A., On the centred Hausdorff measure, J. London Math. Soc. (2) 62 (2000), 843851.

26. Schief, A., Separation properties for self-similar sets, Proc. Amer. Math. Soc. 122 (1994), 111-115.

27. Sullivan, D., Entropy, Hausdorff measures old and new, and limit sets of geometrically finite Kleinian groups, Acta Math. 153 (1984), 259-277.

28. Taylor, S. J., and Tricot, C., Packing measure and its evaluation for a Brownian path, Trans. Amer. Math. Soc. 288 (1985), 679-699.

29. Tricot, C., Two definitions of fractional dimension, Math. Proc. Cambridge Philos. Soc. 91 (1982), 57-74.

30. Tricot, C., Rectifiable and Fractal Sets, pp. 367-403 in: Fractal Geometry and Analysis, Proc. Montreal 1989, Nato Adv. Sci. Inst. (C) 346, Kluwer, Dordrecht 1991. 
31. Zhiwei, Z., and Zuoling, Z., The Hausdorff centred measure of the symmetry Cantor sets, Approx. Theory Appl. 18 (2001), 49-57.

32. Zhou, Z., and Feng, L., A new estimate of the Hausdorff measure of the Sierpinski gasket, Nonlinearity 13 (2000), 479-491.

DEPARTAMENTO DE ANÁLISIS ECONÓMICO: ECONOMÍA CUANTITATIVA

UNIVERSIDAD AUTÓNOMA DE MADRID

CAMPUS DE CANTOBLANCO

28049 MADRID

SPAIN

E-mail: m.1lorente@uam.es
DEPARTAMENTO DE ANÁLISIS ECONÓMICO I UNIVERSIDAD COMPLUTENSE DE MADRID CAMPUS DE SOMOSAGUAS

28223 MADRID

SPAIN

E-mail:mmoranca@ccee.ucm.es 